\title{
Student capabilities to utilize m-learning service in new smart devices
}

\author{
Amir Dirin ${ }^{1}$ Marko Nieminen ${ }^{2}$ Mirka Kettunen ${ }^{3}$ \\ ${ }^{1}$ Haaga-helia University of Applied Science, Helsinki, Finland \\ ${ }^{2}$ Aalto Universities, Konemiehentie 2, Espoo, Finland \\ ${ }^{3}$ Haaga-helia University of Applied Science, Helsinki, Finland
}

\begin{abstract}
A typical gadget that students often carry is a smart phone. Each smart phone brand has often unique features and capabilities. This study reveals how students perceive these devices when they start-up for the first time and discusses the major gaps among these devices based on students' usability experiments. The results help to understand the mobile learning applications usage and demand in smartphones. There have already been many initiatives to enhance mobile phones in education activities but failed to reach a common development framework standard [1].
\end{abstract}

Keywords: Mobile learning, Usability Test, Mobile Usability

\subsection{Introduction}

In this paper we investigate students' expectations and capabilities to utilize mlearning application with different smart phones. Smartphones are inseparable gadgets among students nowadays [2, 3]. This study investigates the usage of smart phones and their applications by users who do not have prior experience on the particular smartphone platform. Our study reveals challenges that students may encounter when using the same mo- bile application such as calendar, browsers and email in different mobile brands. Additionally, the study examines the smoothness of new application installation in different smart phones.

Technology has always been part of educational institutes and assist students and teachers in their educational activities. In recent years, the use of ICT in the education and training has yield several paradigm shifts [4]. The persuasion of technology advancement onto educational process was not only computers or the internet, mobile devices such as tablets and smart phones have significant contributions. We are entering the mobile age, the mobile devices especially mobile phones with its popularity among learners and its portability provide unique opportunity to enhance in education [4]. Utilizing mobile learning in education process has engaged researchers for many years [6]. Usability considerations have been a key issue in mobile learning application development. In this context, usability analysis addresses two paths: 1. Traditional usability testing of mobile learning applications [7, 8, 9] and 2. Pedagogical usability testing of mobile learning solutions [10, 11]. Furthermore, the high penetration among students and advanced functionality provide possibilities both for practitioners and researchers to enhance the use of smartphones in educational process. 


\subsection{User profile and test steps}

The target users in this research were students at Haaga-Helia University of Applied Science. A total of 12 students were chosen among students who had participated in the usability course during fall 2012. The selection was mainly based on students' prior knowledge in using a specific smartphone operating system. Six test users were designated to carry out the experiments on each brand [12]. As there were insufficient qualified test users in the class, some test users were requested to conduct the test twice.

The selected testing devices were Galaxy S II (Android), Apple iPhone 4s (iOS) and Nokia Lumia 800 (Windows) operating system. The test users in each group consisted of users who have not had previous experience in using smartphones and advanced users who had owned a smart device for more than three years. There were 4 female and 8 male test users who were on their first semester up to last semesters of their studies. The age range of the users was 21-31 years.

The user studies were conducted in three different phases. The first phase a webbased questionnaire, the second was a usability test in the usability lab of Aalto University, which is fully equipped for running usability tests. The test users' performance and the smart device screen were monitored and recorded from the control room. The third phase of the study was conducted at Haaga-Helia University of Applied Science media lab. Similarly, all the steps and actions were recorded for further analysis.

\subsection{Applied User Studies Methods}

The web based questionnaire [13] about test users' background addressed following topics: How old are you? Are you male or female? How many years of experience you have in using computers?
Do you own a smart phone? Which mobile operating systems have you experience so far (Android, iOS and Windows Phone)? What Smartphone features / applications are you using?

In the second phase of the study the users were asked to participate in actual usability test sessions at usability lab in Aalto University. Usability testing [14] is a method to identify possible errors and failures. For the usability testing we prepared several tasks that users have to execute during the test session. The tasks were composed based on users' background knowledge on mobile phones' operating system and previous device model experiences. Each student was given 10 minutes time to perform seven independent tasks. The objectives of the usability study were: 1 . To measure the number of tasks the user carries in 10 minutes given time. 2. To determine how smoothly user is able to search, download and use the new applications from application store. 3 . To study how users' prior knowledge on pre-installed applications' experience such as calendar, email etc. would support in new smart phones usage. In usability test session, the tasks were piled and user was asked to carry out one by one.

In the new phone test experiment, user had to start-up the device and go through the device settings tasks without having prior knowledge about the test device. After that, applications were tested: camera, calendar, Wi-Fi settings, internet browser (to navigate predefined tasks); search for predefined application to download from applications store, navigation application usage task. Finally, the users were asked to setup an email account and send an email to predefined email address. Test users were asked to think aloud while performing the tasks. Picture 1 presents the test use cases that users carry out during the test sessions. 


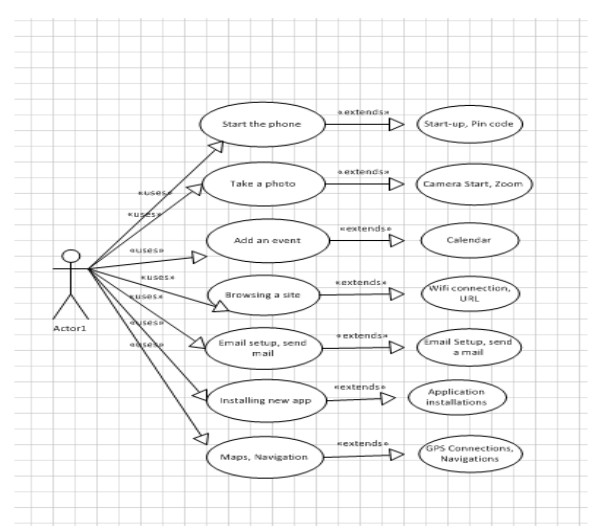

Picture 1. Use cases for the new phone experiment.

After each test session the test user was requested to participate in a short interview. In the interview, user shared his/her test experiences with interviewer. The questions addressed following issues: What went wrong in your opinion? What challenges have you had with the device? Did you have any difficulty to download an application? Did the applications (calendar, mail etc) behave similarly as your own device? Anything else you would like to share?

\section{Research Findings}

The results of this research can help us to learn students' expectations and capabilities on new smart phones and its applications setups. This leads to understanding of a usable mobile learning application demands from students' viewpoint [15]. The analysis of the web based questionnaire data indicates that our test users have approximately 5 years of experience in computer usage. Almost $90 \%$ of test users currently own a smartphone. From all the test users 38\% have experience with Android devices, 54\% have experience in $\mathrm{iOS}$ and $8 \%$ have experience with Windows Phone. The data also shows that all test users have experience either with iOS, Android or Windows and none had prior experience with two or more mobile operating systems. Table 1 summarizes students' mobile application usage. Internet-browser is the most utilized application among students followed by email, calendar, and camera.

\begin{tabular}{|l|c|c|c|c|c|c|c|}
\hline & $\mathbf{0}$ & $\mathbf{1}$ & $\mathbf{2}$ & $\mathbf{3}$ & $\mathbf{4}$ & $\mathbf{5}$ & Avearge \\
\hline Calendar & 0 & 2 & 0 & 0 & 6 & 5 & 3,92 \\
\hline Camera & 0 & 0 & 1 & 4 & 3 & 5 & 3,92 \\
\hline E-mail & 1 & 1 & 1 & 0 & 2 & 8 & 3,92 \\
\hline Internet -browser & 0 & 0 & 1 & 1 & 2 & 9 & 4,46 \\
\hline Navigation & 1 & 0 & 3 & 4 & 1 & 4 & 3,23 \\
\hline Web Search & $\mathbf{1}$ & $\mathbf{1}$ & $\mathbf{2}$ & $\mathbf{0}$ & $\mathbf{4}$ & $\mathbf{5}$ & 3,54 \\
\hline Total & $\mathbf{3}$ & $\mathbf{4}$ & $\mathbf{8}$ & $\mathbf{9}$ & $\mathbf{1 8}$ & $\mathbf{3 6}$ & \\
\hline
\end{tabular}

Table 1. Target group smart phones features usage

The comparative usability evaluation [16] is applied to analyze the usability test data. The data from usability test sessions and post test interview reveals that test users are specifically unhappy with the slowness of the device operating system at the start-up. Additionally, the recorded data unveils that almost all test users have difficulties in setting up the phone for the first time. Moreover, the high sensibility of the touch screen and the small UI elements (keypads) were the third major dissatisfaction factor. For the 10 minutes time to perform a pile of seven tasks, it took 8.81 minutes to complete all tasks with Android, 9.36 minutes with Windows phone and more than 10 minutes with iOS.

In Android phone the test users had difficulties in start up phase due to the slowness of the operating system. The users had also problems in downloading the Gmail application: the download was terminated automatically without any prior notifications. This is considered a serious usability defect as users should be notifying clearly for any failure or errors [15]. The data also shows that users had difficulties in finding the application store on the device that they had no prior experience with. Additionally, the data analysis discloses that users got frustrated with the frequent update notifications pop-up during usability test, and there were no indications regarding the cancellation of 
the notifications. Finally, the data reveals that some users had problems to switch back to home screen from the applications.

Similarly as with Android, the iOS users had difficulties with the slow boot-up of iOS. The test session record shows that after entering the pin code, it took a long time before the home screen gets ready for user interactions. This contradicts with the fact that the product should support the user to work effectively [17]. According to results, the test users had less difficulty in finding an application from the iOS application store than from the Android application store. Aligned with this, the test users also experienced less difficulty in downloading and installing new applications in iOS. Also, searching or applications Windows application store was troublesome for some users. The name Market place was not indicative and clear about the possibility to download new applications. Many users also had problems to navigate between applications in Windows phone.

The test users had difficulties to locate the start-up button in Windows phone. The on/off button is relatively small which is almost hidden at the top of the phone. Additionally, the button required a long press to start up. This was the delay factor on the Windows phone start-up setting phase. The touch screen sensibility of the Windows phone was considered a problem because it resulted in erroneous key presses, which contradicts with Nielsen's rule that the unintentional error should be minimized [17]. A good design anticipates any typing errors [15].

In general, the test users showed their satisfaction specifically with smart phones' touch screen size: it eases the reading and writing on mobile device. The results from the post-test interview indicate that - despite the problems - the test users appreciate the offering of applications through the application stores. However, they were dissatisfied with the inconsistency on the downloading process in different brands and the naming of the application store in the user interface. The usability record analysis shows that users had difficulties in their first attempts to use the pre-installed applications (e.g. calendar, email) in different devices. This difficulty occurred because of the inconsistency on the application implementations in different smart phones.

\section{Results and Conclusions}

The results in this study are valid for the device groups and brands that are mentioned on the previous sections. Moreover, the result is dependent on the users' capabilities as well as the context in which the usability test was conducted. Results may vary depending on the smart devices and the users' previous experience on smartphone usage. Nevertheless, this study complies with the previous research results on smart phone penetrations among students $[2,3]$. Almost $90 \%$ of the test users own a smartphone that use their device constantly.

Internet browser was the most utilized application by users. They use this application to search information during the lectures and other educational activities outside of the schools. Many attempts have already been made to utilize the mobile browser for learning activities e.g. [18]. All test users who owned a smart phone had already customized their device email application with their university email account.

One of the objectives of this research was to measure the students' capabilities to adapt to a new mobile operating system. This is reflected in the students' ability to search and download new application in an environment without previous experience. The lack of earlier experience on 
new operating systems yields to failures and confusions among test users. This indicates that the inconsistency on the smart device operating system construct avoidable confusions if the user intends to change the device brand. The test result also depicts that the inconsistency on the application stores' title in different brands affect the searching and downloading new applications outcomes in the test. Furthermore the descriptive name of the intended application in the application store may speed up the search and downloading process. This recommends that the name of the mobile learning application subjected for students download has to be consistent across platforms as well as carefully selected.

The third objective of this study was to determine whether users previous experience on pre-installed applications such as calendar, email etc. affect the use of similar applications in the new devices. The outcomes of this study convey that having experience with one operating system may not necessarily support the use of the other operating system in an efficient way. As an example, each device has distinct default touch screen sensibility setups. Even though a user may have previous experience with touch screen devices it may not support a positive experience for the startup of the other devices from other brands. Likewise, this study shows that having prior knowledge in mobile applications still results confusions among students with the device of an unfamiliar brand. The inconsistencies of the preinstalled applications in the user interface of different brands, navigation schemes, as well as the look and feel of the applications yield result in confusions.

\section{Discussions and Future Work}

A major finding from our study is that the inconsistencies across smartphone plat- forms create difficulties for users. It is somewhat difficult to overcome this problem since each brand has independent development and user interface requirements and guidelines for developing new applications. However, this trend is changing quickly as all mobile brands are going to support HTML5 applications. This trend supports the conclusion that HTML5-based mobile learning application can overcome the current confusions for the mobile application usage among students. As is seen from the results, the Internet browser was the most frequent used application by all students. Developing application in HTML5 prevail the current applications fragmentation in different brands. All brands and operating system users can therefore use the same look and feel for mobile learning application and create a consistent user experience across devices and platforms.

\section{References}

[1] Mostakhdemin-Hosseini Ali, Tuimala, Jarno "Mobile Learning Framework" International association for development of Information Society, July 17-19 2005, Qawra, Malta.

[2] Maged NK Boulos, Steve Wheeler, Carlos Tavares and Ray Jones. How smartphones are changing the face of mobile and participatory healthcare:an overview, with example from eCAALYX. Retrieved 18.03.2013 from http://www.biomedical-engineeringonline.com/content/10/1/24.

[3] Mostakhdemin-Hosseini Ali, Najafabadi Naghmeh "The Mobile phone Constitutive Effect on Students life in Finland" IEEE-IMCL, Interactive Mobile \& Computer Aided Learning, 19-21 April 2006 Amman-Jordan.

[4] Bransford, J, D., Brown, A. L. \& Cocking, R:R: (1999). How people learn: Brain, Mind, Experience, and School, Na- 
tional Academy press, Washington, D.C. 1998, USA.

[5] Sharples, Mike, “ Big Issues in mobile learning", Report of the workshop by the Kaleidoscope Network of Excellence Mobile learning Initiative, Edited by Mike Sharples, University of Nottingham, 2006.

[6] Mostakhdemin-Hosseini Ali, Mustajärvi Jari, "Steps Required Develop Mobile Learning Services", International association for development of Information Society, July $17-19^{\text {th }}$, 2004. Avila, Spain.

[7] Seong, Su Kuen, Daniel. "Usability guidelines for designing mobile learning portals", Mobile 06 Proceedings of the 3rd international conference on Mobile technology Applications \& Systems ACM New York, NY, USA, 2006 ISBN:1-59593-519-3.

http://portal.acm.org/citation.cfm?id=129 2359

[8] Zhang, D. and Adipat, B. "Challenges, methodologies, and issues in the usability testing of mobile application "International Journal of Human-Computer", 2005

[9] Mostakhdemin-Hosseini Ali, "Usability Considerations of Mobile Learning Applications" June 2009 International Journal of Interactive Mobile Technologies (iJIM) Volume 3, Special Issue 1.

[10] Kukulska-Hulme, Agnes., "Mobile Usability in Educational Contexts: What have we learnt?" International Review of Research in Open and Distance Learning, Volume 8, Number 2. 2007

[11] Mostakhdemin-Hosseini Ali., "Analysis of Pedagogical Considereations of M-learning in smart devices" International Journal of Interactive Mobile Technologies (iJIM) Volume 3, Special Issue 1. 2008.

[12] Nielsen, J. "Why you only need to test with 5 users". Jakob Nielsen's Alertbox: Current Issues in Web Usability. 2000. http://www.units.muohio.edu/mtsc/usabili tytestingrevisedFINAL.pdf

[13] Jansen Karen J., Corely G. Kevin and Jansen Bernard J. "E-survey Methodology", The Pennsylvania State University, 2007, USA.

http://faculty.ist.psu.edu/jjansen/academic /pubs/esurvey chapter_jansen.pdf

[14] Joseph S.Dumas, Janice C. Redish. A practical guide to usability testing. Intellect Books, ISBS, 5804 N.E Hassalo St, Portland, Orange 97213-3644, 1999, USA

[15] Mostakhdemin-Hosseini Ali, Mustajärvi Jari, "Evaluation of Mobile Learning System Prototype", International association for development of Information Society, IADIS Feb $23-25^{\text {th }}, 2005$. Algarve, Portugal.

[16] Molich R., Ede M. R., Kaasgaard K., Karyukin, B. (2004), Comparative usability evaluation, Behavior and Information Technology, 23(1), 65-74.)

[17] Nielsen, Jakob., "Heuristic evaluation", In Nielsen, J. and Mark, R.L.(Eds.) 'Usability Inspection Methods', New York: John Wiley \& Sons. 1994

[18] Daniel Schön, Melanie Klinger, Stephan Kopf, Wolfgang Effelsberg MobileQuiz - "A Lecture Survey Tool using Smartphones and QR Tags"

International Journal of Digital Information and Wireless Communications (IJDIWC) 2(3): 231-244 